\title{
ENGULFING CONTINUA IN AN $n$-CELL
}

\author{
BY \\ RICHARD J. TONDRA( $\left.{ }^{(}\right)$
}

\begin{abstract}
In this paper it is shown that there exist open connected subsets $D_{1}$, $D_{2}$, and $D_{3}$ of an $n$-cell $E$ such that, if $C$ is any proper compact connected subset of $E$ and $C \subset U, U$ open, then there exists a homeomorphism $h$ of $E$ onto itself such that $C \subset h\left(D_{i}\right) \subset U$ for some $i, 1 \leqq i \leqq 3$.
\end{abstract}

1. Introduction. The main results of this paper are the following two theorems:

ENgulfing TheOREM. Let $E$ be an $n$-cell, $n \geqq 2$. There exist proper domains ( $a$ domain is an open, connected subset) $D_{1}, D_{2}$, and $D_{3}$ of $E$ such that if $X \subset E$ is a proper continuum (a continuum is a compact, connected subset) and $G$ is an open set, $X \subset G \subset E$, then there is a homeomorphism $h$ of $E$ onto itself which is isotopic to the identity and

(i) $X \subset h\left(D_{1}\right) \subset G, h \mid$ bd $E=$ identity, if $X \subset$ int $E$;

(ii) $X \subset h\left(D_{2}\right) \subset G$, if $X \cap$ bd $E \neq \varnothing$ and $X \cap$ bd $E \neq$ bd $E$; and

(iii) $X \subset h\left(D_{3}\right) \subset G, h \mid \mathrm{bd} E=$ identity, if $X \cap$ bd $E=\mathrm{bd} E$.

ApProximation TheOrem. Let $E$ be an $n$-cell, $n \geqq 2$. There exists a continuum $C \subset E$ with the following properties:

(i) $C$ is homeomorphic to the 1-point compactification of a connected n-manifold with boundary;

(ii) if $D$ is a proper domain of $E$, then $D=\bigcup_{k=1}^{\infty} C_{k}$, where, for all $k \geqq 1$, $C_{k} \subset \mathrm{i} C_{k+1}$ (i $C_{k+1}$ denotes the point set interior of $C_{k+1}$ relative to $E$ ) and $C_{k}$ is homeomorphic to $C$; and

(iii) if $X$ is a proper continuum in $E$, then $X=\bigcap_{k=1}^{\infty} C_{k}$, where, for all $k, C_{k+1} \subset \mathrm{i} C_{k}$ and $C_{k}$ is homeomorphic to $C$.

In what follows, certain notational conventions and definitions will be used. If $X$ is a topological space and $A$ a subset of $X$, then $\mathrm{i}_{X} A, \operatorname{cl}_{X} A, \operatorname{fr}_{X} A$, and $\operatorname{ed}_{X} A$ will denote the interior, closure, frontier, and edge of $A$ in $X$ respectively, where $\operatorname{ed}_{X} A=A-\mathrm{i}_{X} A$. If there is no possibility of ambiguity, then the subscript " $X$ " will be omitted. If $M$ is a manifold (all manifolds are assumed to be separable

Received by the editors March 31, 1969.

AMS 1969 subject classifications. Primary 5701, 5478.

Key words and phrases. Engulfing, continua, collaring, tapered collaring, domain equivalence, compact equivalence, domain rank.

(1) Part of this material comprised part of the author's thesis written under the direction of P. H. Doyle at Michigan State University. 
metric spaces), then int $M$ and bd $M$ will denote the manifold interior and boundary of $M$ respectively. If a space $X$ is homeomorphic to a space $Y$, then this will be denoted by $X \equiv Y$.

\section{Preliminary results.}

Definition 2.1. Let $L$ and $M$ be $n$-manifolds, $n \geqq 1$, such that bd $L \neq \varnothing$ and $L \subset M . L$ is called a relative $M$ manifold if

(i) $L \cap$ bd $M \neq \varnothing$,

(ii) $L \cap$ bd $M$ is an (n-1)-manifold, and

(iii) ed $L=L-\mathrm{i} L$ is empty or an $(n-1)$-manifold.

Note that if $L$ is a relative $M$ manifold, then bd $(L \cap$ bd $M)=\operatorname{bd}(\operatorname{ed} L)$.

Definition 2.2. Let $L$ and $M$ be $n$-manifolds, $n \geqq 1$, such that $L \subset M$, bd $L \neq \varnothing$, and ed $L \neq \varnothing$. If $L \subset$ int $M$ or if $L$ is a relative $M$ manifold, then $L$ is said to be collared in $M$ provided there is an embedding $h$ of the pair (ed $L \times[0,1)$, (ed $L \cap$ bd $M) \times[0,1)$ ) into the pair $(M$, bd $M$ ) such that

(i) $h(x, 0)=x, x \in$ ed $L$, and

(ii) $h($ ed $L \times[0,1)) \cap L=h($ ed $L \times 0)$.

The set $h($ ed $L \times[0,1))$ is called a collar of $L$ in $M$ and is denoted by $c L$. The set $C L=L \cup c L$ is called a collaring of $L$ in $M$.

Lemma 2.3. Let $L$ be a relative $M$ manifold, $n \geqq 2$, such that ed $L \neq \varnothing$. Then there is an embedding $h$ of the pair ( $\mathrm{ed} L \times[0,1),(\mathrm{ed} L \cap$ bd $M) \times[0,1))$ into $(M, \mathrm{bd} M)$ such that

(i) $h(\mathrm{ed} L \times[0,1)) \subset L$, and

(ii) $h(x, 0)=x, x \in$ ed $L$.

Proof. Let $E=\operatorname{ed} L$. If bd $E=\varnothing$, then, since $E \subset$ bd $L$, the result follows from the main result of [2]. Now suppose that bd $E \neq \varnothing$ and let $Q=L \cap$ bd $M$. Then bd $E=$ bd $Q$. It follows from [2] that there is an embedding $g_{1}:$ bd $E \times[-1,0] \rightarrow E$ such that $g_{1}($ bd $E \times[-1,0])=F_{1}$ is closed in $E$ (that $g_{1}$ can be chosen so that $F_{1}$ is closed is shown possible in the proof of Theorem 2.6) and $g_{1}(x, 0)=x$ for all $x \in$ bd $E$. Also, there is an embedding $g_{2}:$ bd $E \times[0,1) \rightarrow Q$ such that $g_{2}(x, 0)=x$ for all $x \in$ bd $E=$ bd $Q$. Let $P=E \cup g_{2}($ bd $E \times[0,1))$. Since $P \subset$ bd $L$, there is an embedding $g_{3}: P \times[0,1) \rightarrow L$ such that $g_{3}(x, 0)=x, x \in P$, and $g_{3}(P \times[0,1)) \cap$ bd $L$ $=g_{3}(P \times 0)$. Let $f$ be a homeomorphism of bd $E \times([-1,0] \times[0,1))$ onto bd $E \times([-1,1) \times[0,1))$ such that

(i) $f$ restricted to (bd $E \times-1 \times[0,1)) \cup($ bd $E \times[-1,0] \times 0)$ is the identity, and

(ii) $f$ carries bd $E \times 0 \times[0,1)$ homeomorphically onto bd $E \times[0,1) \times 0$.

Define $g$ : bd $E \times[-1,1) \rightarrow$ bd $L$ by

$$
\begin{aligned}
g(x, t) & =g_{1}(x, t), & & t \in[-1,0], \\
& =g_{2}(x, t), & & t \in[0,1) .
\end{aligned}
$$

Then $h_{1}=(g$, id $) f\left(g_{1}^{-1}, \mathrm{id}\right)$, where id is the identity map on $[0,1)$, is a homeomorphism of $\left(g_{1}(\right.$ bd $\left.E \times[-1,0])\right) \times[0,1)$ onto $(g(b d E \times[-1,1)) \times[0,1)$. Let 
$F_{1}=g_{1}(\mathrm{bd} E \times[-1,0])$ and $F_{2}=E-g_{1}(\mathrm{bd} E \times(-1,0])$. Then $F_{1}$ and $F_{2}$ are closed in $E$ and $F_{1} \cap F_{2}=F=g_{1}($ bd $E \times-1)$. Since $h_{1} \mid(F \times[0,1))$ is the identity, the map $h_{2}: E \times[0,1) \rightarrow P \times[0,1)$ defined by

$$
\begin{aligned}
h_{2}(x, t) & =h_{1}(x, t), & & x \in F_{1}, \\
& =(x, t), & & x \in F_{2},
\end{aligned}
$$

is an onto homeomorphism. Since $h_{1}$ carries $g_{1}($ bd $E \times 0) \times[0,1)$ homeomorphically onto $g($ bd $E \times[0,1)) \times 0$ and is the identity on $g_{1}($ bd $E \times 0) \times 0, h=g_{3} h_{2}$ is the required embedding of $(\mathrm{ed} L \times[0,1),(\operatorname{ed} L \cap$ bd $M) \times[0,1))$ into $(M$, bd $M)$.

THEOREM 2.4. Let $L$ and $M$ be $n$-manifolds, $n \geqq 2$, such that $L$ is a closed subset of $M$ and ed $L \neq \varnothing$. Then $L$ is collared in $M$ if

(i) $L \subset$ int $M$ and $P=M$-int $L$ is an $n$-manifold, or

(ii) $L$ is a relative $M$ manifold and $P=M-$ int $L$ is a relative $M$ manifold.

Proof. If (i) holds, then ed $L \subset$ bd $P$ and the result follows from [2]. If (ii) holds, then ed $L=\operatorname{fr} L=\operatorname{fr} P=\operatorname{ed} P$. Since ed $L=\operatorname{ed} P$, the result follows by applying Lemma 2.3 to $P$.

Let $X$ be a metric space, $A$ a subset of $X$, and $f, g: A \rightarrow R$ bounded continuous functions such that, for all $a \in A, f(a) \leqq g(a)$. Let

$$
P[f, g ; A]=\{(x, t) \in X \times R \mid x \in A \text { and } f(x) \leqq t \leqq g(x)\} .
$$

If $f(a)<g(a)$ for all $a \in A$, let

$$
T P[f, g ; A]=\{(x, t) \in X \times R \mid x \in A \text { and } f(x) \leqq t<g(x)\} .
$$

Definition 2.5. Suppose that $L$ is collared in $M$ and that $B$ is a closed set, perhaps empty, $B \subset \mathrm{cl}_{M}\left(\operatorname{ed}_{M} L\right)=C$. Consider ed $L \times[0,1)$ as contained in $C \times R$ and let $h$ : ed $L \times[0,1) \rightarrow M$ give a collar of $L$ in $M$. Let $f$ be continuous, $f: C \rightarrow\left[0, \frac{1}{2}\right)$ such that $f(c)=0$ if and only if $c \in(B \cup(C-\mathrm{ed} L))$. Let $T L$ and $T L^{*}$ be defined by

$$
T L=L \cup h\left(T P\left[0, \frac{1}{2} f ; \text { ed } L-B\right]\right)
$$

and

$$
T L^{*}=L \cup C \cup h\left(P\left[0, \frac{1}{2} f ; \operatorname{ed} L\right]\right) .
$$

$T L$ is called a tapered collaring of $L$ in $M$ with base $B$ and support $F$ if, given an open set $G, L-B \subset G$, then there is a homeomorphism $g$ of $M$ onto itself, which is isotopic to the identity under an isotopy $H$ such that

(i) $L-B \subset g(T L) \subset G$,

(ii) if $L \cup C \subset G$, then $L \cup C \subset g\left(T L^{*}\right) \subset G$, and

(iii) $H(x, t)=x$ for all $x \in(L \cup(M-F)), t \in[0,1]$.

THEOREM 2.6. Let $L$ and $M$ be $n$-manifolds, $L \subset M, L$ a relative $M$ manifold or $L \subset$ int $M$. If $L$ is collared in $M$ and $B$ is a closed set, $B \subset \mathrm{cl}_{M}\left(\operatorname{ed}_{M} L\right)=C$, then $L$ has 
a tapered collaring $T L$ in $M$ with base $B, T L \equiv \mathrm{i}_{M} L$ and $T L^{*} \equiv L \cup C$. Furthermore, if $C$ is compact, then the support $F$ of $T L$ may be chosen to be compact also.

Proof. The proof is straightforward but tedious, and so only an outline of the proof will be given. Let $A=\operatorname{ed}_{M} L$. If $\operatorname{cl}_{M} A$ is compact, let $X=M$; otherwise, let $X$ be the 1-point compactification of $M$ with compactification point $p$, and consider $M$ as embedded in $X$. In either case, since $A$ is locally compact, $D=\mathrm{cl}_{X} A-A$ is either empty or compact. If $B$ is closed, $B \subset \operatorname{cl}_{M}\left(\operatorname{ed}_{M} L\right)=C$, then $B \cup D$ is closed in $E=\mathrm{cl}_{X} A$. Let $h: A \times[0,1) \rightarrow M$ give a collar $c L$ of $L$ in $M$. Since $L$ is a relative $M$ manifold or $L \subset$ int $M$, it follows from Lemma 2.3 or [2] that there is an embedding $h_{1}:(A \times(-1,0],(A \cap$ bd $M) \times(-1,0]) \rightarrow(M$, bd $M) \cap L$ such that $h(x, 0)=h_{1}(x, 0)=x, x \in A$. Since $B \cup D$ is closed in $E$ there exist continuous functions $f: E \rightarrow\left[0, \frac{1}{2}\right)$ and $f_{1}: E \rightarrow\left(-\frac{1}{2}, 0\right]$ such that $f(e)=f_{1}(e)=0$ if and only if $e \in B \cup D$. Let $P=P\left[f_{1}, f ; E\right]$. Then $P$ is compact and there is an embedding $h_{2}: P \rightarrow X$ defined by

$$
\begin{aligned}
h_{2}(x, t) & =x, & & x \in E, t=0, \\
& =h(x, t), & & x \in A, 0 \leqq t \leqq f(x), \\
& =h_{1}(x, t), & & x \in A, f_{1}(x) \leqq t \leqq 0 .
\end{aligned}
$$

Let $G \subset M, G$ open, and suppose that $L-B \subset G$. Since $G$ is open in $M, G$ is open in $X$ and $H=(G-(B \cup D))$ is open in $X$. Let $H^{\prime}=\left(h_{2}\right)^{-1}(H)$. Then $H^{\prime}$ is open in $P$, $(E-(B \cup D)) \times 0 \subset H^{\prime}$, and $(B \cup D) \times 0 \subset P-H^{\prime}$. Therefore there exist continuous functions $g: E \rightarrow\left[0, \frac{1}{2}\right)$ and $g_{1}: E \rightarrow\left(-\frac{1}{2}, 0\right]$ such that

(i) $f_{1}(e) \leqq g_{1}(e) \leqq 0 \leqq g(e) \leqq f(e), e \in E$;

(ii) $f_{1}(e)<g_{1}(e)<0<g(e)<f(e), e \in(E-(B \cup D))$; and

(iii) $P\left[g_{1}, g ; E-(B \cup D)\right] \subset H^{\prime}$.

Let $E_{1}=E$ if $X=M$ and let $E_{1}=E-p$ otherwise. If $L \cup C \subset G$, then $g$ may be chosen so that $P\left[0, \mathrm{~g} ; E_{1}\right] \subset h_{2}^{-1}(G)$. Using the results of $[8, \mathrm{p} .556]$, it is easily established that

(i) $T L=L \cup h\left(T P\left[0, \frac{1}{2} f\right.\right.$; ed $\left.\left.L-B\right]\right)$ is a tapered collaring in $M$ with base $B$;

(ii) $T L \equiv \mathrm{i}_{M} L$; and

(iii) $T L^{*}=h_{2}\left(P\left[0, \frac{1}{2} f ; E_{1}\right) \cup L=L \cup C \cup h\left(P\left[0, \frac{1}{2} f\right.\right.\right.$; ed $\left.\left.L\right]\right)$ is homeomorphic to $L \cup C$.

3. Proof of the main results. The proof of the main results depends upon some results of piecewise linear manifold theory and the results of the previous section. The terminology that will be used is essentially that used by Zeeman in [10] but is modified to agree with the terminology used by Zeeman and Hudson in [6]. By a simplicial complex $K$ of $R^{p}, p \geqq 1$, is meant a locally finite complex consisting of at most a countable number of rectilinear simplexes in a Euclidean $p$-space $R^{p}$. As usual, $|K|$ denotes the polyhedron determined by $K$. An $n$-manifold $M$ is called 
combinatorial or piecewise linear, denoted by $\mathrm{PL}$, if there is a homeomorphism $f$ of $|K|$ onto $M$ where $K$ is a complex in $R^{p}$ such that if $n \geqq 1$ then $|l k(v, K)|$ is a combinatorial $(n-1)$-sphere or $(n-1)$-ball for each vertex $v$ of $K$. The pair $(K, f)$ is called a $P L$ triangulation of $M$. Let $X \subset M, X$ a manifold. If there is a subcomplex $L$ of $K$ such that $(L, f|| L \mid)$ is a PL triangulation of $X$, then we will say that $(K, f)$ restricted to $X$ gives a PL triangulation of $X$. An $m$-manifold $L$ which is embedded as a subset of a PL $n$-manifold $M$ is called $P L$ in $M$ if there is a subdivision $K^{\prime}$ of $K$ and a subcomplex $P^{\prime}$ of $K^{\prime}$ such that $\left(P^{\prime}, f|| P^{\prime} \mid\right)$ is a PL triangulation of $f\left(\left|P^{\prime}\right|\right)=L$. Let $M$ and $N$ be PL manifolds with PL triangulations $(f, K)$ and $(g, L)$ respectively. A continuous function $h: M \rightarrow N$ is called PL if there are subdivisions $K^{\prime}$ of $K$ and $L^{\prime}$ of $L$ such that $g^{-1} h f$ is a simplicial map. If $t$ is an $n$-simplex, let $|t|$ denote the polyhedron $|s(t)|$ where $s(t)=\{s: s$ is a face of $t\}$.

LeMma 3.1. Let $M$ be a PL n-manifold, $n \geqq 2$, and let $L$ be a PL $n$-manifold in $M$, ed $L \neq \varnothing$. If

(i) $L \subset$ int $M$ or

(ii) $L \cap$ bd $M=Q$ is a PL (n-1)-manifold in $M$, then $L$ is collared in $M$.

Proof. The result is easily established and the proof is only outlined. The first step is to use the method employed (with proper modifications in case $L \cap$ bd $M$ is a PL $(n-1)$-manifold in $M)$ in the proof of Lemma 17 of Chapter 3 of [10] to show that $M$-int $L$ is a PL $n$-manifold in $M$. If (ii) holds, then the above method shows that bd $M$-int $Q$ is empty or a PL $(n-1)$-manifold in $M$ and that bd $L$ -int $Q=\operatorname{ed} L=$ ed $(M-$ int $L)$ is a PL $(n-1)$-manifold in $M$. Thus both $L$ and $M$-int $L$ are relative $M$ manifolds. Since $L$ is $\mathrm{PL}$ in $M, L$ is closed and it follows from Theorem 2.4 that $L$ is collared in $M$.

LEMMA 3.2. Let $M$ be a connected $P L$-manifold, $n \geqq 2$, with $P L$ triangulation $(K, f), D$ a proper domain of $M$, and $C$ a continuum contained in $D$. Then there exists a compact, connected, $P L$ n-manifold $L$ in $M$ such that

(i) $C \subset$ i $L \subset L \subset D, L \neq D$;

(ii) $L=f(|P|)$ where $P$ is a subcomplex of $\mathrm{Sd}_{j} K$, the jth regular barycentric subdivision of $K$, for some $j \geqq 1$;

(iii) $L \subset$ int $M$ if $C \subset$ int $M$; and

(iv) $L \cap$ bd $M=L \cap$ bd $D$ is a $P L(n-1)$-manifold if $C \cap$ bd $M \neq \varnothing$.

Proof. Since $K$ is locally finite, it may be assumed that $M$ is embedded in some $R^{p}$ as a closed subset and that $M$ has PL triangulation $(K, \mathrm{id})$, where id denotes the identity map. Let $d$ be the Euclidean metric on $R^{p}$ restricted to $M$ and choose $\rho>0$ such that $0<\rho \leqq d(C, M-D)$ and set $Q=\{x \in M: d(x, C)<\rho\}$. Note that $Q \subset D$ and that if $C \cap$ bd $M=\varnothing$ then it may be assumed that $\rho$ is chosen so that $Q \subset$ int $M$. The existence of $L$ is now established using the results and terminology of [6]. 
If $J$ is a simplicial complex, $X \subset|J|$, let $N(X, J)=\{s \in J \mid s$ is a face of $t, t \cap X \neq \varnothing\}$. Let $L_{1}=N(C, K)$. Since $C$ is compact, $L_{1}$ is finite and $C \subset \mathrm{i}_{M}\left|L_{1}\right|$. There exists an integer $q \geqq 1$ such that if $L_{2}=N\left(C, \mathrm{Sd}_{q} L_{1}\right)$, then, for all $j \geqq q, N\left(\left|L_{2}\right|, \mathrm{Sd}_{j} L_{1}\right)$ $=N\left(\left|L_{2}\right|, \mathrm{Sd}_{j} K\right)$ and $\left|N\left(\left|L_{2}\right|, \mathrm{Sd}_{j} L_{1}\right)\right| \subset Q$. If $C \cap$ bd $M \neq \varnothing$, then there is an $n$-simplex $t_{n} \in L_{2}$ such that $t_{n}$ has an $(n-1)$-face $t_{n-1}$ contained in bd $M$. Let $b$ denote the barycenter of $t_{n-1}$. Then there exists an $n$-simplex $t_{n}^{\prime} \in \operatorname{Sd}_{2} L_{2}$ such that $b \in\left|t_{n}^{\prime}\right|,\left\{t_{n}^{\prime} \mid \cap\right.$ bd $M=\left|t_{n-1}^{\prime}\right|$, where $t_{n-1}^{\prime}$ is an $(n-1)$-face of $t_{n}^{\prime}$, and $\left|t_{n}^{\prime}\right| \subset \mathrm{i}_{M}\left|t_{n}\right|$. If $C \cap$ bd $M \neq \varnothing$, let $R=\operatorname{Sd}_{2} L_{2}-\left\{t_{n}^{\prime}, t_{n-1}^{\prime}\right\}$ and $S=\left\{u \mid u\right.$ is a proper face of $\left.t_{n-1}^{\prime}\right\}$; if $C \cap$ bd $M \neq \varnothing$, let $R=\operatorname{Sd}_{2} L_{2}$ and $S=\varnothing$. In either case, $|R|$ is link collapsible on $|S|$. Furthermore, if $C \cap$ bd $M \neq \varnothing$, then $|R| \cap$ bd $M$ is link collapsible on $|S| \cap$ bd $M=$ bd $\left|t_{n-1}^{\prime}\right|$. Let $J=N\left(|R|-|S|, \operatorname{Sd}_{q+4} K\right)$. Then $J$ is a subcomplex of $N\left(\left|L_{2}\right|, \mathrm{Sd}_{q+4} L_{1}\right)$ and so $|J| \subset Q$. Since $|R|-|S|$ is connected, it follows from Theorem 1 of [6] that $|J|$ is a compact, connected PL $n$-manifold in $M$ such that $|J| \cap$ bd $M=\varnothing$ if $C \cap$ bd $M=\varnothing$, and $|J| \cap$ bd $M$ is a PL $(n-1)$-manifold in $M$ if $C \cap$ bd $M \neq \varnothing$. If $C \cap$ bd $M=\varnothing$, let $L=|J|$; otherwise, let $P=J \cup \operatorname{Sd}_{2} s\left(t_{n}^{\prime}\right)$ and let $L=|P|$. It is easily seen that $L$ is the required PL $n$-manifold in $M$.

For $n \geqq 1$, let $E^{n}=\left\{x=\left(x_{1}, \ldots, x_{n}\right) \in R^{n} \mid\|x\| \leqq 1\right\}, S^{n-1}=\left\{x \in R^{n} \mid\|x\|=1\right\}$ and consider $R^{n-1}, E^{n-1}$, and $S^{n-2}$ as embedded in $R^{n}, E^{n}$, and $S^{n-1}$ respectively in the usual manner. For $n \geqq 2$, define $f: E^{n-1} \rightarrow R$ by $f(x)=\left(1-\sum_{k=1}^{n-1} x_{k}^{2}\right)^{1 / 2}$. Then $f$ is continuous and $f(x)=0$ if and only if $x \in S^{n-2}$. For each $t \in[0,1]$, let $B^{n}(t)$ $=\left\{(x, s) \mid x \in E^{n-1}\right.$ and $\left.0 \leqq s \leqq t f(x)\right\}, A^{n}(t)=\left\{(x, s) \in B^{n}(t) \mid x_{1} \geqq 0\right\}$ and $F^{n-1}(t)$ $=\left\{(x, s) \in A^{n}(t) \mid x_{1}=0\right\}$. The following result follows easily from [8] and the proof is omitted.

LeMma 3.3. Let $0<s<t<1$. There is a homeomorphism $h[s, t]$ of $B^{n}(1)$ onto itself such that

(i) $h[s, t]\left(B^{n}(t)\right)=B^{n}(s)$;

(ii) $h[s, t] \mid$ bd $B^{n}(1)=$ identity;

(iii) $g[s, t]=h[s, t] \mid A^{n}(1)$ is a homeomorphism of $A^{n}(1)$ onto itself carrying $A^{n}(t)$ onto $A^{n}(s)$ and $g[s, t]$ restricted to $\left(\mathrm{bd} B^{n}(1) \cap A^{n}(1)\right)=$ identity;

(iv) $h[s, t]$ is isotopic to the identity relative to bd $B^{n}(1)$ and $g[s, t]$ is isotopic to the identity relative to bd $B^{n}(1) \cap A^{n}(1)$.

If $X$ is a topological space and $Y \subset X$, let $I(X)$ rel $Y=\{h \mid h$ is a homeomorphism of $X$ which is isotopic to the identity relative to $Y$ \} (if $Y=\varnothing$, we will write $I(X)$ ) and let $C I(X)=\{h \mid h$ is a homeomorphism of $X$ onto itself which is isotopic to the identity under an isotopy which is the identity outside some proper compact subset of $X$ \}.

DefinITION 3.4. Let $k_{j}, 1 \leqq j \leqq n$, be integers. A homeomorphism $h$ of $R^{n}$ onto itself is called an integral translation if $h$ is defined by

$$
h\left(x_{1}, \ldots, x_{n}\right)=\left(x_{1}+k_{1}, \ldots, x_{n}+k_{n}\right) .
$$


Definition 3.5. Let $I^{n}$ denote the unit cube and consider $I^{n}$ as embedded in $R^{n}$. A PL triangulation ( $K$, id) of $R^{n}$ is called an integral PL triangulation if

(i) $\left(K\right.$, id) restricted to $I^{n}$ is a PL triangulation of $I^{n}$ and

(ii) any integral translation of $R^{n}$ induces a PL isomorphism of $K$ onto itself.

It is easily established that integral PL triangulations exist for $R^{n}$. Let $H^{n}=\left\{x \in R^{n} \mid x_{n} \geqq 0\right\}$. If ( $K$, id) (id denotes the identity homeomorphism) is an integral PL triangulation of $R^{n}$, then $\left(K\right.$, id) restricted to $H^{n}$ is a PL triangulation of $H^{n}$ which will be denoted by $(L$, id). If $(K$, id) is an integral PL triangulation of $R^{n}$, then clearly $\left(\mathrm{Sd}_{j} K, \mathrm{id}\right)$ is also for all $j \geqq 1$.

Henceforth, let $\left(K\right.$, id) denote a fixed integral PL triangulation of $R^{n}$ and $(L$, id) the restriction of $\left(K\right.$, id) to $H^{n}$. Let

$$
\begin{array}{r}
M(K)=\left\{P \mid P \text { is a subcomplex of some } \operatorname{Sd}_{j} K, j \geqq 1 \text {, and }|P|\right. \text { is a } \\
\text { compact, connected, PL } \left.n \text {-manifold in } R^{n}\right\}
\end{array}
$$

and

$M(L)=\left\{P \mid P\right.$ is a subcomplex of some $\operatorname{Sd}_{j} K, j \geqq 1,|P|$ is a compact, connected PL $n$-manifold in $H^{n}$ which is a relative $H^{n}$ manifold, and $|P| \cap$ bd $H^{n}$ is a PL $(n-1)$-manifold in $\left.H^{n}\right\}$.

Lemma 3.6. Let $P \in M(K)$. There exists $h \in C I\left(R^{n}\right)$ such that $h(|P|)=\left|P^{\prime}\right|$ for some $P^{\prime} \in M(L)$.

Proof. Let $q$ be an integer such that $|P| \subset$ int $X, X=\left\{x \in R^{n} \mid x_{n} \geqq q\right\}$. Since $\left(K\right.$, id) is an integral PL triangulation of $R^{n},(K$, id) restricted to $X$ is a PL triangulation $(Q$, id) of $X$. Since $|P| \subset$ int $X$, there exists a PL $n$-cell $B$ in $Q$ such that $B=|J|$, $J$ a subcomplex of some $\operatorname{Sd}_{j} Q, j \geqq 1$, and $B \cap|P|$ is a PL $(n-1)$-cell in $X$ contained in bd $|P|$ and $B \cap$ bd $X$ is a PL $(n-1)$-cell in $X$. Let $P^{*}=\operatorname{Sd}_{j} P \cup J$. Then $P^{*}$ is a subcomplex of $\operatorname{Sd}_{j} Q$. It follows from Theorem 7, Chapter 3 of [10] that there exists $f \in C I\left(R^{n}\right)$ such that $f(|P|)=\left|P^{*}\right|$. Since $\left|P^{*}\right| \cap$ bd $X$ is a PL $(n-1)$-manifold in $X$, it follows that the integral translation $g: R^{n} \rightarrow R^{n}$ defined by $g\left(x_{1}, \ldots, x_{n}\right)$ $=\left(x_{1}, \ldots, x_{n}-q\right)$ carries $\left|P^{*}\right|$ onto $\left|P^{\prime}\right|, P^{\prime} \in M(L)$. Therefore there exists $g^{\prime} \in C I\left(R^{n}\right)$ such that $g^{\prime}\left(\left|P^{*}\right|\right)=\left|P^{\prime}\right|$. Then $h=g^{\prime} f \in C I\left(R^{n}\right)$ is the required homeomorphism.

LEMMA 3.7. There exists a continuum $C \subset H^{n} \subset R^{n}, n \geqq 2$, with the following properties:

(i) $C$ is homeomorphic to the 1-point compactification of a connected n-manifold with boundary;

(ii) there exists $C_{1}$ and $D_{1}, C_{1} \equiv C, D_{1}$ a connected n-manifold without boundary, $D_{1}=\mathrm{i}_{R^{n}} C_{1} \equiv \mathrm{i}_{R^{n}} C$ such that if $F_{1}$ is a continuum in $R^{n}$ and $U$ is open in $R^{n}, F_{1} \subset U$, then there exists $g \in C I\left(R^{n}\right)$ such that $F_{1} \subset g\left(D_{1}\right) \subset g\left(C_{1}\right) \subset U$; and

(iii) there exists $C_{2}$ and $D_{2}, C_{2} \equiv C, D_{2}$ a connected n-manifold with boundary, 
$D_{2}=\mathrm{i}_{H^{n}} C_{2} \equiv \mathrm{i}_{H^{n}} C$ such that if $F_{2}$ is a continuum in $H^{n}, F_{2} \cap$ bd $H^{n} \neq \varnothing$, and $V$ is open in $H^{n}, F_{2} \subset V$, then there exists $h \in C I\left(H^{n}\right)$ such that $F_{2} \subset h\left(D_{2}\right) \subset h\left(C_{2}\right) \subset V$.

Proof. If $F_{1}$ is a continuum in $R^{n}$ and $F_{1} \subset U, U$ open in $R^{n}$, then it follows from Lemma 3.2 that there exists $P \in M(K)$ such that $F_{1} \subset|P| \subset U$. Similarly if $F_{2}$ is a continuum in $H^{n}, F_{2} \cap$ bd $H^{n} \neq \varnothing$, and $F_{2} \subset V, V$ open in $H^{n}$, there exists $Q \in M(L)$ such that $F_{2} \subset|Q| \subset V$. Therefore it suffices to establish the result for continua of the form $F_{1}=|P|, P \in M(K)$ and $F_{2}=|Q|, Q \in M(L)$.

Let $(K, \mathrm{id})$ be the given fixed integral PL triangulation of $R^{n},(L, \mathrm{id})$ the restriction of $(K, \mathrm{id})$ to $H^{n},(R, \mathrm{id})$ a fixed PL triangulation of $U^{n}=\left\{x \in R^{n} \mid x_{1}>0\right\}$, and (S, id) a fixed PL triangulation of $V^{n}=\left\{x \in H^{n} \mid x_{1}>0\right\}$. For each integer $j \geqq 1$, let $c(j)=\left\{x \in R^{n} \mid x_{1}=1 / j\right\}$, and for $i, j \geqq 1, i<j$, let $s l(j, i)=\left\{x \in R^{n} \mid 1 / j \leqq x_{1} \leqq 1 / i\right\}$. Set $q=(1,0, \ldots, 0), p=(0,0, \ldots, 0), A=1 \times[-1,1] \times \cdots \times[-1,1]$, where $[-1,1]$ appears $(n-1)$ times, and $B=\left\{x \in A \mid x_{n} \geqq 0\right\}$. Let $E$ be the convex hull of $p \cup A$ and $F$ the convex hull of $p \cup B$. Then $E$ is a PL $n$-cell in $R^{n}, F$ is a PL $n$-cell in $H^{n}$, and $F \cap$ bd $H^{n}=G$ is a PL $(n-1)$-cell in $H^{n}$. Let $X^{*}=\left\{R^{n}, H^{n}, U^{n}, V^{n}\right\}$. Note that if $N \subset R^{n}$ is a compact $m$-manifold, and $N$ is PL in $X, X \in X^{*}$, then $N$ is PL in $Y$ for any $Y \in X^{*}$ such that $X \subset Y$. For each $j \geqq 1$, let $E_{j}=E \cap s l(j+1, j)$ and $F_{j}=F \cap \operatorname{sl}(j+1, j)$. Then $E_{j}$ is a PL $n$-cell in $U^{n}$ and $F_{j}$ is a PL $n$-cell in $V^{n}$ and $F_{j} \cap$ bd $H^{n}$ is a PL $(n-1)$-cell in $V^{n}$. We now begin the construction of $C$. Let $\left\{Q_{j}\right\}_{j=1}^{\infty}$ be an enumeration of $M(L)$. For each $j \geqq 1$, there exists a PL homeomorphism $l_{j}, l_{j} \in C I\left(H^{n}\right)$, which extends to a PL homeomorphism $k_{j} \in C I\left(R^{n}\right)$ such that $k_{j}\left(\left|Q_{j}\right|\right)=M_{j}, M_{j} \subset \mathrm{i}_{H^{n}} F_{j} \subset \mathrm{i}_{R^{n}} E_{j}$. Then $M_{j}$ is PL in $V^{n}$. Let $W$ denote the unit $n$-cube, $W=[0,1] \times \cdots \times[0,1], X=\left\{w \in W \mid w_{n}=0\right\}, \quad Y=\left\{x \in W \mid w_{n}=1\right\}$, and $Z=\left\{w \in W \mid w_{n-1}=1\right\}$. By recursive construction a sequence $\left\{f_{j}\right\}_{j=1}^{\infty}$ of embeddings of $W$ in $V^{n}$ can be constructed such that for $j \geqq 1$

(i) $\left\{f_{j}(W)\right\}_{j=1}^{\infty}$ is a disjoint collection of PL $n$-cells in $V^{n}$;

(ii) $f_{j}(W) \cap$ bd $H^{n}=f_{j}(Z)=Z_{j}$ is a PL $(n-1)$-cell in $V^{n}$;

(iii) $f_{j}(W) \subset\left(\mathrm{i}_{H^{n}}\left(F_{j} \cup F_{j+1}\right)\right) \cap\left(\mathrm{i}_{R^{n}}\left(E_{j} \cup E_{j+1}\right)\right)$;

(iv) $f_{j}(W) \cap F_{j}=G_{j}$ is a PL $n$-cell in $V^{n}$ and $f_{j}(W) \cap F_{j+1}=H_{j+1}$ is a PL $n$-cell in $V^{n}$;

(v) $G_{j} \cap$ bd $H^{n}=G_{j}^{\prime}$ is a PL $(n-1)$-cell in $V^{n}$ and $H_{j+1} \cap$ bd $H^{n}=H_{j+1}^{\prime}$ is a PL $(n-1)$-cell in $V^{n}$;

(vi) $f_{j}(W) \cap c(j+1)=A_{j}$ is a $\mathrm{PL}(n-1)$-cell in $V^{n}$ and $A_{j} \cap$ bd $H^{n}=B_{j}$ is a PL $(n-2)$-cell in $V^{n}$;

(vii) $f_{j}(W) \cap M_{j}=f_{j}(X)=X_{j}$ is a PL $(n-1)$-cell in $V^{n}, X_{j} \subset \operatorname{ed}_{H^{n}} M_{j}$, and $X_{j} \cap$ bd $H^{n}=X_{j}^{\prime}$ is a PL $(n-2)$-cell in $V^{n}$;

(viii) $f_{j}(W) \cap M_{j+1}=f_{j}(Y)=Y_{j+1}$ is a PL $(n-1)$-cell in $V^{n}, Y_{j+1} \subset \operatorname{ed}_{H^{n}} M_{j+1}$, and $Y_{j+1} \cap$ bd $H^{n}=Y_{j+1}^{\prime}$ is a PL $(n-2)$-cell in $V^{n}$.

Let $M=\left(\bigcup_{j=1}^{\infty} M_{j}\right) \cup\left(\bigcup_{j=1}^{\infty} f_{j}(W)\right)$. Then it is easily seen that $M$ is a PL $n$-manifold in both $U^{n}$ and $V^{n}$, and $M \cap$ bd $V^{n}=M \cap$ bd $H^{n}$ is a PL $(n-1)$ manifold in $V^{n}$. Therefore it follows from Lemma 3.1 that $M$ is collared in $U^{n}$ and 


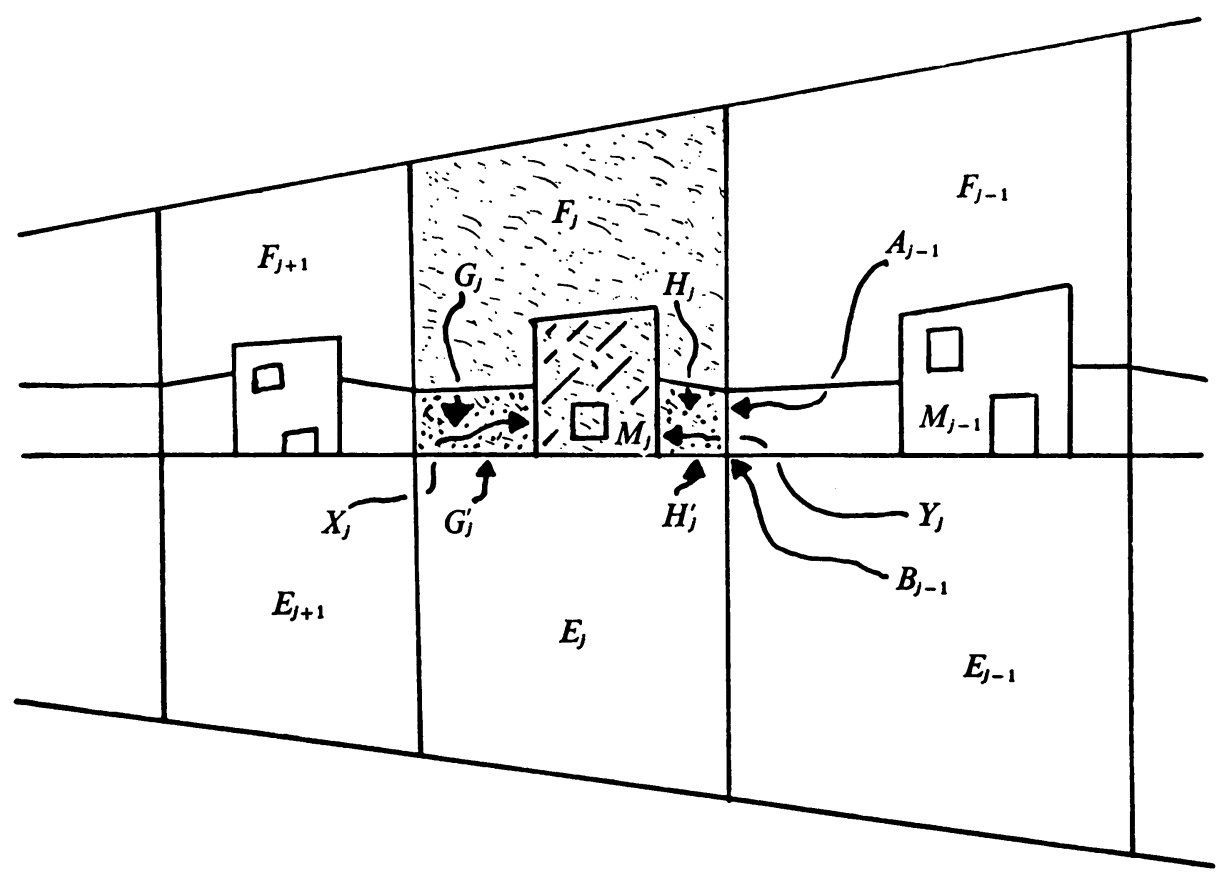

Figure 1

$V^{n}$ and hence collared in $R^{n}$ and $H^{n}$. Since $\operatorname{cl}_{R^{n}}\left(\operatorname{ed}_{R^{n}} M\right)=\operatorname{ed}_{R^{n}} M \cup p$ and $\operatorname{cl}_{H^{n}}\left(\operatorname{ed}_{H^{n}} M\right)=\operatorname{ed}_{H^{n}} M \cup p$ are both compact, it follows from Theorem 2.6 that there exists a tapered collaring $T M_{1}$ of $M$ in $R^{n}$ with base $p$ and a tapered collaring $T M_{2}$ of $M$ in $H^{n}$ with base $p$. Let $D_{1}=T M_{1}, D_{2}=T M_{2}, C_{1}=T M_{1}^{*}, C_{2}=T M_{2}^{*}$, and $C=\mathrm{cl}_{R^{n}} M=\mathrm{cl}_{H^{n}} M=M \cup p$. Then it follows from Theorem 2.6 that $C_{1} \equiv C \equiv C_{2}$, $D_{1} \equiv \mathrm{i}_{R^{n}} C$, and $D_{2} \equiv \mathrm{i}_{H^{n}} C$. For $k>1$ define

$$
\begin{aligned}
& T_{k}^{1}=f_{k-1}(W) \cup\left(\bigcup_{q=1}^{k-1} F_{q}\right)=H_{k} \cup\left(\bigcup_{q=1}^{k-1} F_{q}\right), \\
& T_{k}^{2}=f_{k}(W) \cup\left(\bigcup_{q=k+1}^{\infty} F_{q}\right) \cup p=G_{k} \cup\left(\bigcup_{q=k+1}^{\infty} F_{q}\right) \cup p, \\
& N_{k}=R^{n}-\mathrm{i}_{R^{n}} M_{k}, \text { and } \\
& P_{k}=H^{n}-\mathrm{i}_{H^{n}} M_{k} .
\end{aligned}
$$

Then for $k>1$

(i) $T_{k}^{1}, T_{k}^{2}$ are PL $n$-cells in $H^{n}$;

(ii) $T_{k}^{1} \cap$ bd $H^{n}=L_{k}^{1}, T_{k}^{2} \cap$ bd $H^{n}=L_{k}^{2}$ are PL $(n-1)$-cells in $H^{n}$;

(iii) $T_{k}^{1}, T_{k}^{2}$ are relative $N_{k}$ and $P_{k}$ manifolds, $T_{k}^{1} \cap M_{k}=T_{k}^{1} \cap$ bd $M_{k}=f_{k-1}(Y)$ $=Y_{k}$ and $T_{k}^{2} \cap M_{k}=T_{k}^{2} \cap$ bd $M_{k}=f_{k}(X)=X_{k}$ are PL $(n-1)$-cells contained in bd $N_{k}$ and bd $P_{k}$; and

(iv) $L_{k}^{1} \cup Y_{k}=T_{k}^{1} \cap$ bd $P_{k}, L_{k}^{2} \cup X_{k}=T_{k}^{2} \cap$ bd $P_{k}$, and $L_{k}^{1} \cap Y_{k}, L_{k}^{2} \cap X_{k}$ are PL $(n-2)$-cells in $P_{k}$. 
Therefore it follows from Theorem 2.6 that there exist embeddings $\alpha_{1}, \alpha_{2}: B^{n}(1) \rightarrow N_{k}$ such that

(i) $\alpha_{1}\left(B^{n}(1)\right) \cap \alpha_{2}\left(B^{n}(1)\right)=\varnothing$,

(ii) $\alpha_{q}\left(B^{n}(0)\right)=T_{k}^{q} \cap$ bd $N_{k}=\alpha_{q}\left(B^{n}(1)\right) \cap$ bd $N_{k}, q=1,2$,

(iii) $\alpha_{q}\left(B^{n}(1)\right) \cap M_{k}=\alpha_{q}\left(B^{n}(0)\right), q=1,2$, and

(iv) $\alpha_{q}\left(B^{n}\left(\frac{1}{2}\right)\right)=T_{k}^{q}, q=1,2$;

and embeddings $\beta_{1}, \beta_{2}:\left(A^{n}(1), F^{n-1}(1)\right) \rightarrow\left(P_{k}, P_{k} \cap\right.$ bd $\left.H^{n}\right)$ such that

(v) $\beta_{1}\left(A^{n}(1)\right) \cap \beta_{2}\left(A^{n}(1)\right)=\varnothing$,

(vi) $\beta_{q}\left(A^{n}(0)\right)=T_{k}^{q} \cap M_{k}, q=1,2$,

(vii) $\beta_{q}\left(A^{n}(1)\right) \cap M_{k}=\beta_{q}\left(A^{n}(0)\right), q=1,2$,

(viii) $\beta_{q}\left(A^{n}\left(\frac{1}{2}\right)\right)=T_{k}^{q}, q=1,2$, and

(ix) $\beta_{q}\left(A^{n}(1)\right) \cap$ bd $H^{n}=\beta_{q}\left(F^{n-1}(1)\right), q=1,2$.

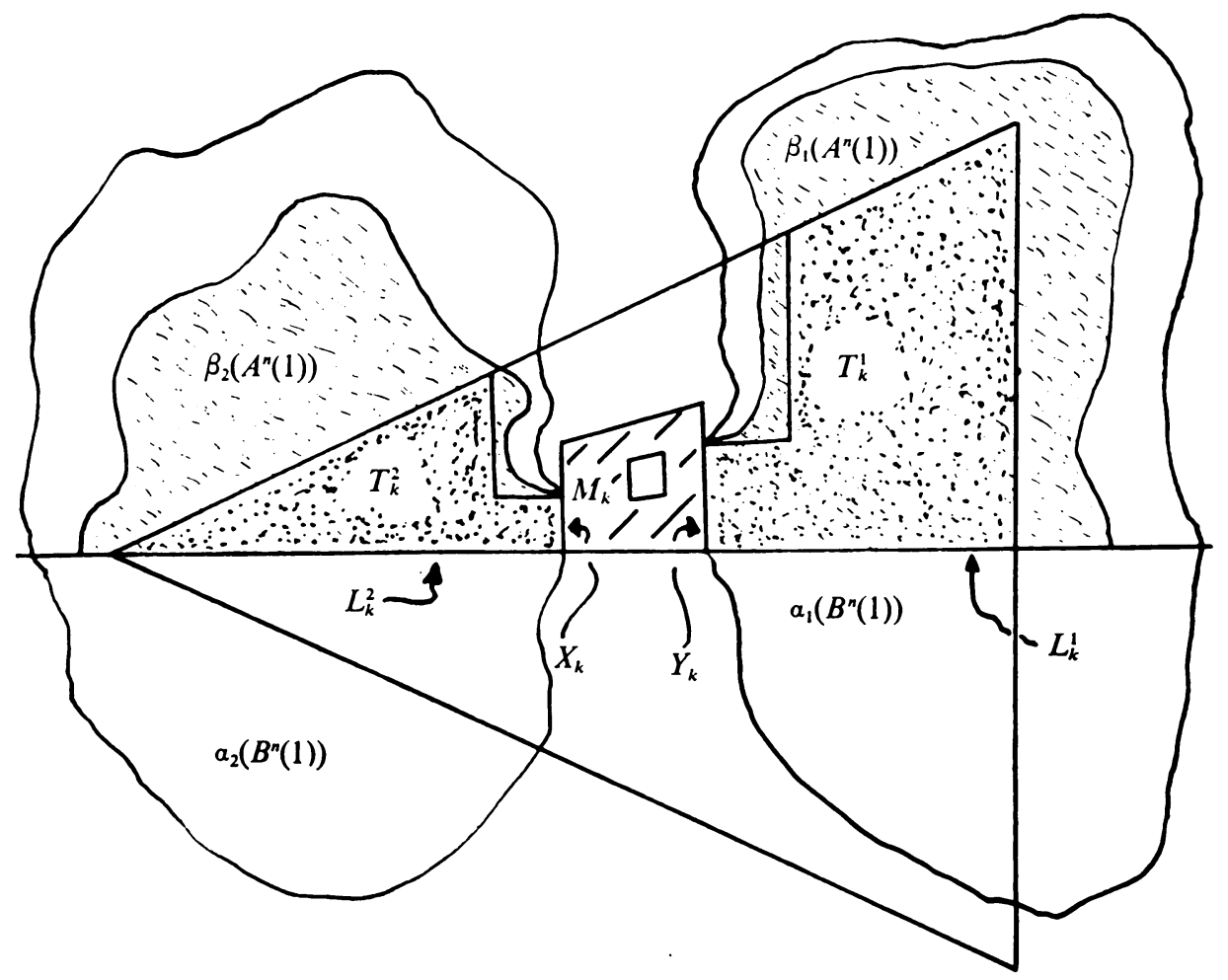

FIGURE 2

Note that $C \subset M_{k} \cup T_{k}^{1} \cup T_{k}^{2}$.

Suppose that $P \in M(K)$ and that $U$ is open in $R^{n},|P| \subset U$. It follows from Lemma 3.6 that there exists $h \in C I\left(R^{n}\right)$ such that $h(|P|)=\left|Q_{k}\right|, Q_{k} \in M(L)$. Since $\mathrm{Sd}_{j} Q_{k} \in M(L), j \geqq 1$, we may assume that $k>1$. Let $k_{k} \in C I\left(R^{n}\right)$ be the previously chosen PL homeomorphism with the property that $k_{k}\left(\left|Q_{k}\right|\right)=M_{k}$. Then 
$h_{k}=k_{k} h \in C I\left(R^{n}\right), h_{k}(|P|)=M_{k}$, and $M_{k} \subset U_{1}=h_{k}(U)$. Since $U_{1}$ is open in $R^{n}$ and $\alpha_{q}\left(B^{n}(0)\right) \subset U_{1}, q=1,2$, there exists $t \in\left(0, \frac{1}{2}\right)$ such that $\alpha_{q}\left(B^{n}(t)\right) \subset U_{1}, q=1,2$. Define $g_{1}: R^{n} \rightarrow R^{n}$ by

$$
\begin{aligned}
g_{1}(x) & =x, & & x \notin \mathrm{i}_{R^{n}}\left(\alpha_{1}\left(B^{n}(1)\right) \cup \alpha_{2}\left(B^{n}(1)\right)\right), \\
& =\alpha_{q} h\left[t, \frac{1}{2}\right] \alpha_{q}^{-1}(x), & & x \in \alpha_{q}\left(B^{n}(1)\right), \quad q=1,2 .
\end{aligned}
$$

(See Lemma 3.3 for the definition of $h\left[t, \frac{1}{2}\right]$.) Then $g_{1} \in C I\left(R^{n}\right), g_{1}(C) \subset U_{1}$, and $g_{1} \mid M_{k}=$ identity. Let $U_{2}=g_{1}^{-1}\left(U_{1}\right)$; then $U_{2}$ is open in $R^{n}, C \subset U_{2}$. Since $D_{1}$ is a tapered collaring of $M$ in $R^{n}$ with base $p$ and compact support, it follows from Definition 2.5 that there exists $g_{2} \in C I\left(R^{n}\right)$ such that $g_{2} \mid C=$ identity, $C-\{p\} \subset g_{2}\left(D_{1}\right) \subset g_{2}\left(C_{1}\right) \subset U_{2}$. Let $g=h_{k}^{-1} g_{1} g_{2}$; then $g \in C I\left(R^{n}\right)$ and $|P| \subset g\left(D_{1}\right)$ $\subset g\left(C_{1}\right) \subset U$ and thus (ii) of the lemma has been established.

Now suppose that $Q_{k} \in M(L)$ and that $V$ is open in $H^{n},\left|Q_{k}\right| \subset V$. Again we may assume that $k>1$. Let $l_{k} \in C I\left(H^{n}\right)$ be the previously chosen PL homeomorphism with the property that $M_{k}=l_{k}\left(\left|Q_{k}\right|\right)$. Then $M_{k} \subset V_{1}=l_{k}(V), V_{1}$ open in $H^{n}$. Therefore there exists $t \in\left(0, \frac{1}{2}\right)$ such that $\beta_{q}\left(A^{n}(t)\right) \subset V_{1}, q=1,2$. Define $h_{1}: H^{n} \rightarrow H^{n}$ by

$$
\begin{aligned}
h_{1}(x) & =x, & & x \notin \mathrm{i}_{H^{n}}\left(\beta_{1}\left(A^{n}(1)\right) \cup \beta_{2}\left(A^{n}(1)\right)\right), \\
& =\beta_{q} g\left[t, \frac{1}{2}\right] \beta_{q}^{-1}(x), & & x \in \beta_{q}\left(A^{n}(1)\right), \quad q=1,2 .
\end{aligned}
$$

Then $h_{1} \in C I\left(H^{n}\right), h_{1}(C) \subset V_{1}$, and $h_{1} \mid M_{k}=$ identity. Let $V_{2}=h_{1}^{-1}\left(V_{1}\right)$; then $V_{2}$ is open in $H^{n}$ and $C \subset V_{2}$. Since $D_{2}$ is a tapered collaring of $M$ in $H^{n}$ with base $p$ and compact support, it follows from Definition 2.5 that there exists $h_{2} \in C I\left(H^{n}\right)$ such that $h_{2} \mid C=$ identity and $C-\{p\} \subset h_{2}\left(D_{2}\right) \subset h_{2}\left(C_{2}\right) \subset V_{2}$. Let $h=l_{k}^{-1} h_{1} h_{2}$. Then $h \in C I\left(H^{n}\right)$ and $\left|Q_{k}\right| \subset h\left(D_{2}\right) \subset h\left(C_{2}\right) \subset V$ and (iii) of the lemma is established and the proof is complete.

LEMMA 3.8. Let $E$ be an $n$-cell, $n \geqq 2$. There exists $C_{3} \subset E$ such that

(i) $C_{3} \equiv C_{1}$ (see Lemma 3.7 for definition of $C_{1}$ );

(ii) $D_{3}=\mathrm{i}_{E} C_{3}$ is a connected n-manifold and bd $D_{3}=$ bd $E$; and

(iii) if $F$ is a proper continuum, bd $E \subset F$, and $U$ is open in $E, F \subset U$, then there exists $g \in I(E)$ rel bd $E$ such that $F \subset g\left(D_{3}\right) \subset g\left(C_{3}\right) \subset U$.

Proof. It follows from the construction of $M$ in Lemma 3.7 that there exists a sequence $\left\{B_{j}\right\}_{j=2}^{\infty}$ of PL $n$-cells in $R^{n}$ such that for $j \geqq 2$

(i) $B_{j} \cap M=B_{j} \cap$ bd $M$ is a PL $(n-1)$-sphere in $R^{n}$;

(ii) $B_{j} \subset \mathrm{i}_{R^{n}} F_{k(j)}, k(j) \geqq 2$; and

(iii) $k(j+1)>k(j)$.

Let $B_{1}$ be a PL $n$-cell in $R^{n}, B_{1} \subset \mathrm{i}_{R^{n}} M_{1}$. Then it is easily seen that $C-$ int $B_{1} \equiv C$ where $C$ is the continuum defined in Lemma 3.7. Let $f: C \rightarrow C_{1}$ be a homeomorphism and let $B=f\left(B_{1}\right)$ and $C_{3}=f\left(C-\right.$ int $\left.B_{1}\right)=C_{1}-$ int $B$. Then $C_{3} \equiv C$ and bd $B$ is a bicollared (n-1)-sphere in $R^{n}$. Let $S$ denote the 1-point compactification of $R^{n}$ 
with compactification point $q$ and consider $R^{n}$ and all its subsets as subsets of $S$. Let $E=S-$ int $B$, then $E$ is an $n$-cell and $\mathrm{i}_{E} C_{3}=D_{3}$ is a connected $n$-manifold with bd $D_{3}=$ bd $E$. Note that $C_{1}=C_{3} \cup B$ and $D_{1}=D_{3} \cup B$.

Suppose that $F$ is a proper continuum in $E$, bd $E \subset F$, and $F \subset U, U$ open in $E$. Let $F_{1}=F \cup B$ and $U_{1}=U \cup B$; then $F_{1}$ is a continuum in $S$ and $U_{1}$ is open in $S$. There exists $f \in I(S)$ such that $f \mid B=$ identity, $f \mid E \in I(E)$, and $f\left(F_{1}\right)=F^{\prime} \subset R^{n}$. Let $U^{\prime}=f\left(U_{1}\right) \cap R^{n}$; then $U^{\prime}$ is open in $R^{n}$ and $F^{\prime} \subset U^{\prime}$. Suppose that there exists $h^{\prime} \in I(S)$ such that $h^{\prime}(E)=E, h^{\prime}(q)=q, h^{\prime} \mid$ bd $E=$ identity, and $F^{\prime} \subset h^{\prime}\left(D_{1}\right) \subset h^{\prime}\left(C_{1}\right)$ $\subset U^{\prime}$. Let $g^{\prime}=f^{-1} h^{\prime}$; then $g^{\prime}(E)=E, g^{\prime} \mid$ bd $E=$ identity, and $g^{\prime}($ int $B$ ) $=$ int $B$. Since $h^{\prime}($ int $B)=$ int $B$ and $F^{\prime}-$ int $B \subset h^{\prime}\left(D_{1}\right)-$ int $B \subset h^{\prime}\left(C_{1}\right)$-int $B \subset U^{\prime}-$ int $B$, it follows that $F \subset g^{\prime}\left(D_{3}\right) \subset g^{\prime}\left(C_{3}\right) \subset U$. Therefore $g=g^{\prime} \mid E$ satisfies (iii) (the fact that $g \in I(E)$ rel bd $E$ follows from [1]). Therefore it suffices to establish the existence of $h^{\prime}$.

Since $F^{\prime} \subset U^{\prime} \subset R^{n}$, it follows from Lemma 3.7 that there exists $h_{1} \in C I\left(R^{n}\right)$ such that $F^{\prime} \subset h_{1}\left(D_{1}\right) \subset h_{1}\left(C_{1}\right) \subset U^{\prime}$. Therefore $h_{1}$ extends to a homeomorphism $g_{1} \in I(S)$ such that $g_{1}$ is the identity in a neighborhood of $q$. Let $A=\{g \in I(S) \mid g$ is the identity in a neighborhood of $q$ and $g \mid\left(S-g_{1}\left(D_{1}\right)\right)=$ identity\}. Note that if $g \in A$, then $g\left(g_{1}\left(D_{1}\right)\right)=g_{1}\left(D_{1}\right)$ and $F^{\prime} \subset g\left(g_{1}\left(D_{1}\right)\right)=g_{1}\left(D_{1}\right)$. If $g \in I(S)$, then $g(B)$ and $g(E)$ are $n$-cells in $S$ with bicollared boundaries-this fact will be used without explicit mention. Since $B \cup g_{1}(B) \subset g_{1}\left(D_{1}\right)$, there exists $g_{2} \in A$ such that $g_{2} g_{1}(B)$ $\subset$ int $B$. It now follows from Lemma 2 of [3] that there exists $g_{3} \in A$ such that $B \subset$ int $g_{3} g_{2} g_{1}(B)$. Let $g_{4}=g_{3} g_{2}$ and $g_{5}=g_{4} g_{1}$; then $g_{4} \in A$ and $F^{\prime} \subset g_{5}\left(D_{1}\right) \subset U^{\prime}$. Let $\alpha_{1}$ : bd $E \times[1,2] \rightarrow E$ be such that $\alpha_{1}(x, 1)=x, x \in$ bd $E$, and $\alpha_{1}($ bd $E \times[1,2])$ $\subset D_{1}$. Define $\beta_{1}$ : bd $E \times[1,2] \rightarrow g_{5}(E)$ by $\beta_{1}=g_{5} \alpha_{1}$. Since $g_{5}$ is the identity in a neighborhood of $q, \rho$ : bd $E \rightarrow S$ defined by $\rho(x)=\beta_{1}(x, 1)$ and $j$ : bd $E \rightarrow S$ defined by $j(x)=x$ have similar orientations (see [4, p. 3]). Therefore it follows from Theorem 3.5 of [4] that there exists a homeomorphism $\beta_{0}:$ bd $E \times[0,1] \rightarrow g_{5}(B)$ - int $B$ such that $\beta_{0}(x, 0)=x$ and $\beta_{0}(x, 1)=\beta_{1}(x, 1), x \in$ bd $E$. Therefore $\beta:$ bd $E \times[0,2] \rightarrow E$ defined by

$$
\begin{aligned}
\beta(x, t) & =\beta_{0}(x, t), & & t \in[0,1], \\
& =\beta_{1}(x, t), & & t \in[1,2],
\end{aligned}
$$

is an embedding into $E$. Furthermore, $B \subset$ int $g_{5}(B) \subset g_{5}\left(D_{1}\right)$ and thus $\beta$ (bd $E \times[0,2]) \subset g_{5}\left(D_{1}\right)$. Let $f$ be a homeomorphism of bd $E \times[1,2]$ onto bd $E \times[0,2]$ such that $f(x, 2)=(x, 2)$ and $f(x, 1)=(x, 0), x \in$ bd $E$. Define $h^{\prime}: S \rightarrow S$ by

$$
\begin{aligned}
h^{\prime}(y) & =y, & & y \in B, \\
& =\beta f \alpha_{1}^{-1}(y), & & y \in \alpha_{1}(\text { bd } E \times[1,2]), \\
& =g_{5}(y), & & y \in E-\alpha_{1}(\operatorname{bd} E \times[1,2)) .
\end{aligned}
$$

Then $h^{\prime}$ is the required homeomorphism. 
4. Proof of the engulfing and approximation theorems. Using the results of $\S 3$, these theorems are easily established.

Proof of the Engulfing Theorem. Let $e$ be an embedding of $R^{n}$ onto int $E, E$ an $n$-cell, and let $e_{1}$ be an embedding of $H^{n}$ onto $E-p, p \in$ bd $E$. Consider $R^{n}$ and $H^{n}$ as subsets of $E$. Let $X$ be a proper continuum, $X \subset G, G$ open in $E$. If $X \subset$ int $E$, then it follows from Lemma 3.7 that there exists $g \in C I\left(R^{n}\right)$ such that $X \subset g\left(D_{1}\right)$ $\subset G \cap R^{n}$. Since $g \in C I\left(R^{n}\right), g$ extends to $h \in I(E)$ rel bd $E$ such that $X \subset h\left(D_{1}\right) \subset G$. If $X \cap$ bd $E$ is a proper subset of bd $E$, there exists $f \in I(E)$ such that $f(X) \subset H^{n}$. It follows from Lemma 3.7 that there exists $g \in C I\left(H^{n}\right)$ such that $f(X) \subset g\left(D_{2}\right)$ $\subset f(G) \cap H^{n}$. Since $g \in C I\left(H^{n}\right), g$ extends to $h^{\prime} \in I(E)$. Let $h=f^{-1} h^{\prime}$. Then $h \in I(E)$ and $X \subset h\left(D_{2}\right) \subset G$. If bd $E \subset X$, the existence of $h \in I(E)$ rel bd $E$ such that $X \subset h\left(D_{3}\right) \subset G$ follows immediately from Lemma 3.8. Thus the theorem is established.

Proof of the Approximation Theorem. If $D$ is a proper domain of $E, E$ an $n$-cell, $n \geqq 2$, then it is easily established that $D=\bigcup_{k=1}^{\infty} C_{k}$, where, for $k \geqq 1, C_{k}$ is a continuum and $C_{k} \subset \mathrm{i}_{E} C_{k+1}$. If $F$ is any proper continuum of $E$, it is easily established that $F=\bigcap_{k=1}^{\infty} C_{k}$ where, for $k \geqq 1, C_{k}$ is a continuum and $C_{k+1} \subset \mathrm{i}_{E} C_{k}$. In view of the above remarks, the proof of the Approximation Theorem follows immediately from the results of the preceding section.

5. Applications to domain rank. If $X$ is a topological space and $U$ an open subset, a topological space $g(U)$ is called a generator of $U$ if $U$ is an open monotone union of $g(U)$; that is $U=\bigcup_{k=1}^{\infty} U_{k}$ where, for all $k \geqq 1, U_{k} \equiv g(U), U_{k} \subset U_{k+1}$, and $U_{k}$ is open in $X$. A set $G$ of connected nonempty topological spaces is called a set of generating domains for $X$ if for each proper domain $D$ of $X$ there exists $G_{\alpha} \in G$ such that $G_{\alpha}$ generates $D$. The domain rank of $X$, denoted by DR $(X)$, is defined by $\operatorname{DR}(X)=\operatorname{glb}\{|A| \mid A$ is a set of generating domains for $X\}$, where $|A|$ denotes the cardinality of the set $A$. A set $B$ of generating domains for $X$ is called basic if $|B|=\mathrm{DR}(X)$. We now give a proof of the result mentioned in [9].

THEOREM 5.1. DR $\left(R^{n}\right)=1, \mathrm{DR}\left(H^{n}\right)=2$, and $\mathrm{DR}\left(E^{n}\right)=3$.

Proof. Clearly $\mathrm{DR}\left(R^{n}\right) \geqq 1, \mathrm{DR}\left(H^{n}\right) \geqq 2$, and $\mathrm{DR}\left(E^{n}\right) \geqq 3$. It follows easily from the results of $\S 3$ that $\left\{D_{1}\right\},\left\{D_{1}, D_{2}\right\}$, and $\left\{D_{1}, D_{2}, D_{3}\right\}$ are basic sets of generating domains for $R^{n}, H^{n}$, and $E^{n}$ respectively.

For $n \geqq 2$, let $F(n)=\{M \mid M$ is a connected $n$-manifold, DR $(M)<\infty\}$. We are interested in defining an equivalence relation in the class $F(n)$ which equates those $n$-manifolds which have a common basic set of generating domains. Let $M$, $N \in F(n) . M$ and $N$ are called domain equivalent, denoted by $M \stackrel{\text { d }}{=}$, if $M$ and $N$ have a common basic set of generating domains. $M$ and $N$ are called compactly equivalent, denoted by $M \stackrel{\mathrm{c}}{=} N$, if

(i) for each proper compact set $C$ of $M$ there is an embedding $h$ of $(C, C \cap$ bd $M)$ into $(N$, bd $N)$ such that $h(C) \neq N$, and 
(ii) for each proper compact set $C$ of $N$ there is an embedding $h$ of $(C, C \cap$ bd $N)$ into $(M$, bd $M)$ such that $h(C) \neq M$.

Clearly $\stackrel{c}{=}$ is an equivalence relation in $F(n)$. The following theorem establishes that $\stackrel{d}{=}$ is also an equivalence relation in $F(n)$ and the relation between $\stackrel{c}{=}$ and $\stackrel{d}{=}$.

THEOREM 5.2. Let $M, N \in F(n) . M \stackrel{\mathrm{c}}{=} N$ if and only if $M \stackrel{\mathrm{d}}{=} N$.

Proof. Suppose that $M \stackrel{\text { d }}{=} N$. If $C$ is a proper compact set of $M$, then since $n \geqq 2$, there exists a proper domain $D$ of $M$ such that $C \subset D$. Since $M$ and $N$ have a common basic set of generating domains, it follows easily that there is an embedding $h$ of $(C, C \cap$ bd $M)$ into $(N$, bd $N)$ such that $h(C) \neq N$. Similarly if $C$ is a proper compact set of $N$, then there is an embedding $h$ of $(C, C \cap$ bd $N)$ into $(M$, bd $M)$ such that $h(C) \neq M$. Therefore $M \stackrel{\mathrm{c}}{=} N$.

Now suppose that $M \stackrel{\mathrm{c}}{=} N$. Let $B=\left\{B_{1}, \ldots, B_{k}\right\}$ be a basic set of generating domains for $M$ and let $G$ be a proper domain of $N$. Since $n \geqq 2$, there exists a sequence $\left\{G_{j}\right\}_{j=1}^{\infty}$ of domains of $N$ such that

(i) $\mathrm{cl}_{N} G_{j}$ is compact, $\mathrm{cl}_{N} G_{j} \subset G, j \geqq 1$;

(ii) $\mathrm{cl}_{N} G_{j} \subset G_{j+1}, j \geqq 1$; and

(iii) $G=\bigcup_{j=1}^{\infty} \mathrm{cl}_{N} G_{j}$.

Since $M \stackrel{\mathrm{c}}{=} N$, for each $j \geqq 2$, there exists an embedding $f_{j}$ of $\left(\mathrm{cl} G_{j}, \operatorname{cl} G_{j} \cap\right.$ bd $\left.N\right)$ into $(M$, bd $M)$ such that $f_{j}\left(\mathrm{cl} G_{j}\right) \neq M$. Then $f_{j}\left(\mathrm{cl} G_{j-1}\right)$ is compact, $f_{j}\left(G_{j}\right)$ is a proper domain of $M$, and $f_{j}^{-1}$ is an embedding of $\left(f_{j}\left(G_{j}\right), f_{j}\left(G_{j}\right) \cap\right.$ bd $\left.M\right)$ into $(N$, bd $N)$. Since $B$ is a basic set of generating domains for $M$, there exists an integer $q(j)$, $1 \leqq q(j) \leqq k$, and an embedding $h_{j}$ of $\left(B_{q(j)}\right.$, bd $\left.B_{q(j)}\right)$ into $(M$, bd $M)$ such that $f_{j}\left(\mathrm{cl} G_{j-1}\right) \subset h_{j}\left(B_{q(j)}\right) \subset f_{j}\left(G_{j}\right)$. Therefore $\mathrm{cl} G_{j-1} \subset f_{j}^{-1} h_{j}\left(B_{q(j)}\right) \subset G_{j}$ and $f_{j}^{-1} h_{j}\left(B_{q(j)}\right)$ is a domain of $N$. Since $|B|$ is finite, it follows that $B$ is a set of generating domains for $N$ and thus DR $(N) \leqq \mathrm{DR}(M)$. Similarly it can be shown that DR $(M) \leqq \mathrm{DR}(N)$. Therefore $B$ is a basic set of generating domains for $N$ and $M \stackrel{\mathrm{d}}{=} N$.

We close noting that if $M$ is a $W$-body (see [7]) which is not embeddable in $R^{3}$, then $M \stackrel{\mathrm{c}}{=} R^{3}$ so $\mathrm{DR}(M)=\mathrm{DR}\left(R^{3}\right)=1$. Thus there exists a domain of $R^{3}$ which generates an open connected 3-manifold which is not embeddable in $R^{3}$.

\section{BIBLIOGRAPHY}

1. J. W. Alexander, On the deformation of an n-cell, Proc. Nat. Acad. Sci. U.S.A. 9 (1923), 406-407.

2. M. Brown, Locally fat embeddings of topological manifolds, Topology of 3-Manifolds and Related Topics (Proc. Univ. of Georgia Inst., 1961), Prentice-Hall, Englewood Cliffs, N. J., 1962, pp. 83-91. MR 28 \#1598.

3. - The monotone union of open n-cells is an open n-cell, Proc. Amer. Math. Soc. 12 (1961), 812-814. MR 23 \#A4129.

4. M. Brown and H. Gluck, Stable structures on manifolds. I. Homeomorphisms of $S^{n}$, Ann. of Math. (2) 79 (1964), 1-17. MR 28 \#1608a.

5. P. H. Doyle and J. G. Hocking, $A$ decomposition theorem for $n$-dimensional manifolds, Proc. Amer. Math. Soc. 13 (1962), 469-471. MR 25 \#4514. 
6. J. F. P. Hudson and E. C. Zeeman, On regular neighborhoods, Proc. London Math. Soc. (3) 14 (1964), 719-745. MR 29 \#4063.

7. D. R. McMillan, Jr., Summary of results on contractible open manifolds, Topology of 3-Manifolds and Related Topics (Proc. Univ. of Georgia Inst., 1961), Prentice-Hall, Englewood Cliffs, N. J., 1962, pp. 100-102. MR 28 \#1601.

8. M. H. A. Newman, The engulfing theorem for topological manifolds, Ann. of Math. (2) 84 (1966), 555-571. MR 34 \#3557.

9. R. J. Tondra, The domain rank of an n-sphere and an n-cell, Notices Amer. Math. Soc. 15 (1968), 940-941. Abstract \#68T-G21.

10. E. C. Zeeman, Seminar on combinatorial topology, Inst. Hautes Études Sci., Paris, 1963 (mimeograph).

Iowa STate University, AMES, IOWA 50010 